\title{
Nodeless superconductivity and time-reversal symmetry breaking in the noncentrosymmetric superconductor $\operatorname{Re}_{24} \mathbf{T i}_{5}$
}

\author{
T. Shang, ${ }^{1,2,3,{ }^{*}}$ G. M. Pang,,${ }^{4,5}$ C. Baines, ${ }^{6}$ W. B. Jiang, ${ }^{4,5}$ W. Xie, ${ }^{4,5}$ A. Wang, ${ }^{4,5}$ M. Medarde,,${ }^{1}$ E. Pomjakushina, ${ }^{1}$ M. Shi, ${ }^{2}$ \\ J. Mesot, ${ }^{7,3,8}$ H. Q. Yuan, ${ }^{4,5, \dagger}$ and T. Shiroka ${ }^{8,7, \dagger}$ \\ ${ }^{1}$ Laboratory for Multiscale Materials Experiments, Paul Scherrer Institut, Villigen CH-5232, Switzerland \\ ${ }^{2}$ Swiss Light Source, Paul Scherrer Institut, Villigen CH-5232, Switzerland \\ ${ }^{3}$ Institute of Condensed Matter Physics, École Polytechnique Fédérale de Lausanne (EPFL), Lausanne CH-1015, Switzerland \\ ${ }^{4}$ Department of Physics, Zhejiang University, Hangzhou 310058, China \\ ${ }^{5}$ Center for Correlated Matter, Zhejiang University, Hangzhou 310058, China \\ ${ }^{6}$ Laboratory for Muon-Spin Spectroscopy, Paul Scherrer Institut, CH-5232 Villigen PSI, Switzerland \\ ${ }^{7}$ Paul Scherrer Institut, CH-5232 Villigen PSI, Switzerland \\ ${ }^{8}$ Laboratorium für Festkörperphysik, ETH Zürich, CH-8093 Zurich, Switzerland
}

(Received 13 November 2017; published 9 January 2018)

\begin{abstract}
The noncentrosymmetric superconductor $\mathrm{Re}_{24} \mathrm{Ti}_{5}$, a time-reversal symmetry- (TRS-) breaking candidate with $T_{c}=6 \mathrm{~K}$, was studied by means of muon-spin rotation/relaxation $(\mu \mathrm{SR})$ and tunnel-diode oscillator techniques. At the macroscopic level, its bulk superconductivity was investigated via electrical resistivity, magnetic susceptibility, and heat-capacity measurements. The low-temperature penetration depth, superfluid density, and electronic heat capacity all evidence an $s$-wave coupling with an enhanced superconducting gap. The spontaneous magnetic fields revealed by zero-field $\mu$ SR below $T_{c}$ indicate a time-reversal symmetry breaking and thus the unconventional nature of superconductivity in $\mathrm{Re}_{24} \mathrm{Ti}_{5}$. The concomitant occurrence of TRS breaking also in the isostructural $\mathrm{Re}_{6}(\mathrm{Zr}, \mathrm{Hf})$ compounds hints at its common origin in this superconducting family and that an enhanced spin-orbital coupling does not affect pairing symmetry.
\end{abstract}

DOI: 10.1103/PhysRevB.97.020502

Superconductors with inversion centers can host either even-parity spin-singlet or odd-parity spin-triplet states. These strict symmetry-imposed requirements, however, are relaxed in noncentrosymmetric superconductors (NCSCs) where paritymixed superconducting states are also allowed. In these materials the lack of an inversion symmetry often induces an antisymmetric spin-orbit coupling (ASOC), which can lift the degeneracy of conduction-band electrons. Since the extent of parity mixing is determined by the strength of the SOC, formally similar compounds, but with different spin-orbit couplings, can exhibit different degrees of parity mixing.

The recent interest in NCSCs is related to the complex nature of their superconducting properties [1,2]. Because of the mixed pairing, noncentrosymmetric superconductors can display significantly different properties compared to their conventional counterparts. Some NCSCs, such as $\mathrm{CePt}_{3} \mathrm{Si}$ [3], $\mathrm{CeIrSi}_{3}$ [4], $\mathrm{Li}_{2} \mathrm{Pt}_{3} \mathrm{~B}$ [5,6], and $\mathrm{Mo}_{3} \mathrm{Al}_{2} \mathrm{C}$ [7] exhibit line nodes, whereas others, such as $\mathrm{LaNiC}_{2}$ [8] and $(\mathrm{La}, \mathrm{Y})_{2} \mathrm{C}_{3}$ [9] show multiple superconducting gaps. Furthermore, because of the spin-triplet pairing, the upper critical field often exceeds the Pauli limit as has been found, e.g., in $\mathrm{CePt}_{3} \mathrm{Si}$ [10] and $\mathrm{Ce}(\mathrm{Rh}, \mathrm{Ir}) \mathrm{Si}_{3}$ [11,12]. Finally, some NCSCs, as e.g., $\mathrm{LaNiC}_{2}$ [13], $\operatorname{Re}_{6}(\mathrm{Zr}, \mathrm{Hf})[14,15]$, and $\mathrm{La}_{7} \mathrm{Ir}_{3}$ [16] are known to break the time-reversal symmetry (TRS).

\footnotetext{
*Corresponding author: tian.shang@psi.ch

†hqyuan@zju.edu.cn

†tshiroka@phys.ethz.ch
}

The binary alloy $\mathrm{Re}_{24} \mathrm{Ti}_{5}$ is a NCSC with superconducting temperature $T_{c}=6 \mathrm{~K}$ as reported already in the 1960s [17], but its macroscopic physical properties were studied in detail only recently [18]. In this Rapid Communication we explore in details the microscopic nature of its superconductivity (SC). Similar to $\mathrm{Re}_{24} \mathrm{Zr}_{5}$ and $\mathrm{Re}_{24} \mathrm{Nb}_{5}, \mathrm{Re}_{24} \mathrm{Ti}_{5}$ also adopts an $(\alpha-\mathrm{Mn})$-type crystal structure with space-group $I-43 m$. However, although the former compounds have been widely studied by means of macro- and microscopic techniques $[19,20]$, much less is known about $\operatorname{Re}_{24} \mathrm{Ti}_{5}$. A simple analogy, based on structural similarity, can lead to the wrong conclusions since a SOC-dependent parity mixing can bring about rather different superconducting properties. Since its sister compounds $\operatorname{Re}_{6}(\mathrm{Zr}, \mathrm{Hf})$ are known to break the TRS in the superconducting state $[14,15], \mathrm{Re}_{24} \mathrm{Ti}_{5}$ represents an ideal opportunity to search for TRS breaking and unconventional SC in a material with a modified SOC value. Moreover, the study of additional NCSCs can bring new insight into the nature of unconventional superconductivity in general.

Considering the key role played by muon-spin-relaxation and rotation $(\mu \mathrm{SR})$ techniques in unraveling the presence of TRS breaking in unconventional superconductors [21], in this Rapid Communication, we report on the systematic magnetization, transport, thermodynamic, tunnel-diode oscillator (TDO), and $\mu \mathrm{SR}$ studies of $\mathrm{Re}_{24} \mathrm{Ti}_{5}$, with particular focus on the latter. We find that below $T_{c}$ spontaneous magnetic fields appear, implying a superconducting state which breaks TRS and has an unconventional nature. The low-temperature 


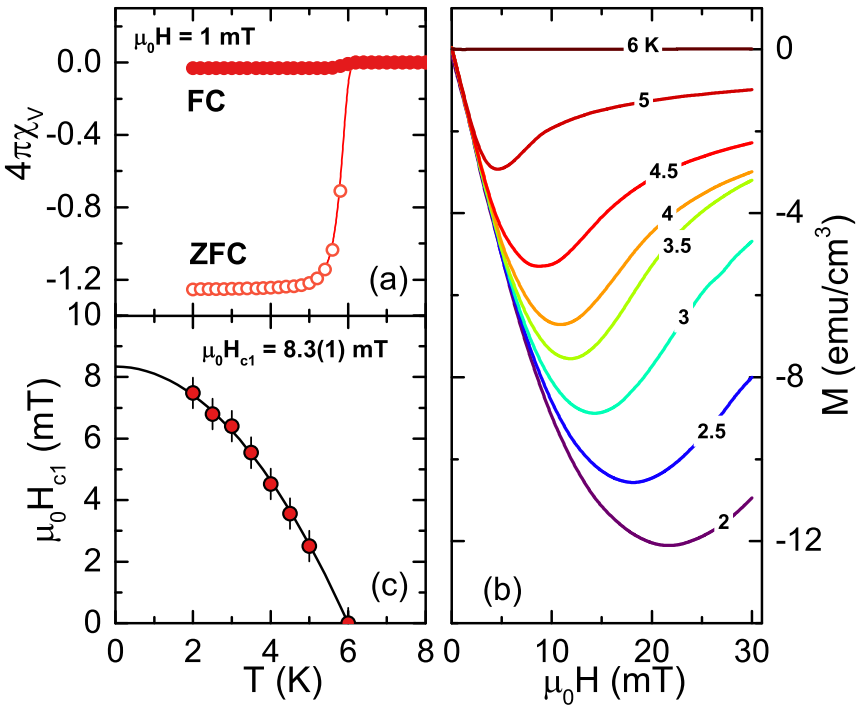

FIG. 1. (a) Temperature dependence of magnetic susceptibility $\chi(T)$ for $\mathrm{Re}_{24} \mathrm{Ti}_{5}$. (b) Magnetization versus applied magnetic field recorded at different temperatures up to $T_{c}$. For each temperature, $\mu_{0} H_{c 1}$ was determined as the value where $M(H)$ deviates from linearity. (c) $\mu_{0} H_{c 1}$ versus temperature: the solid line, a fit to $\mu_{0} H_{c 1}(T)=$ $\mu_{0} H_{c 1}(0)\left[1-\left(T / T_{c}\right)^{2}\right]$, determines $\mu_{0} H_{c 1}(0)=8.3(1) \mathrm{mT}$.

penetration depth, superfluid density, and electronic specific heat all suggest a nodeless $s$-wave pairing mechanism.

Polycrystalline $\mathrm{Re}_{24} \mathrm{Ti}_{5}$ samples were prepared by arc melting $\mathrm{Re}$ and $\mathrm{Ti}$ metals under argon atmosphere and then annealed at $900{ }^{\circ} \mathrm{C}$ for two weeks. The $\mathrm{x}$-ray powder diffraction, measured on a Bruker D8 diffractometer, confirmed the $\alpha$-Mn structure of $\operatorname{Re}_{24} \mathrm{Ti}_{5}$. Magnetic susceptibility, electrical resistivity, and specific-heat measurements in different applied magnetic fields were performed on a 7-T Quantum Design magnetic property measurement system and a 14-T physical property measurement system. The $\mu \mathrm{SR}$ measurements were carried out using the general-purpose instrument located at the $\pi \mathrm{M} 3$ beamline of the Swiss Muon Source of the Paul Scherrer Institut in Villigen, Switzerland. The temperature-dependent shift of magnetic-penetration depth was measured by using a TDO technique in a $\mathrm{He}^{3}$ cryostat at an operating frequency of $7 \mathrm{MHz}$.

The magnetic susceptibility, measured at $1 \mathrm{mT}$ using field-cooling (FC) and zero-field-cooling (ZFC) procedures, is shown in Fig. 1(a). The splitting of the two curves is typical of type-II superconductors, and the ZFC susceptibility indicates bulk superconductivity with $T_{c}=6 \mathrm{~K}$. The electrical resistivity drops at the onset of superconductivity at $6.8 \mathrm{~K}$, becoming zero at $6 \mathrm{~K}$ [see Fig. 2(a)]. The bulk nature of SC is further confirmed by specific-heat data [see Fig. 2(b)].

In transverse-field- (TF-) $\mu \mathrm{SR}$ measurements of superconductors, the applied magnetic field should exceed the lower $\mu_{0} H_{c 1}$ critical value so that the additional fielddistribution broadening due to the flux-line lattice (FLL) can be quantified from the muon decay rate. To determine $\mu_{0} H_{c 1}$, the field-dependent magnetization was preliminarily measured at various temperatures below $T_{c}$ as shown in Fig. 1(b). The derived $\mu_{0} H_{c 1}$ values are plotted in Fig. 1(c) as a function of temperature. The solid line is a fit to

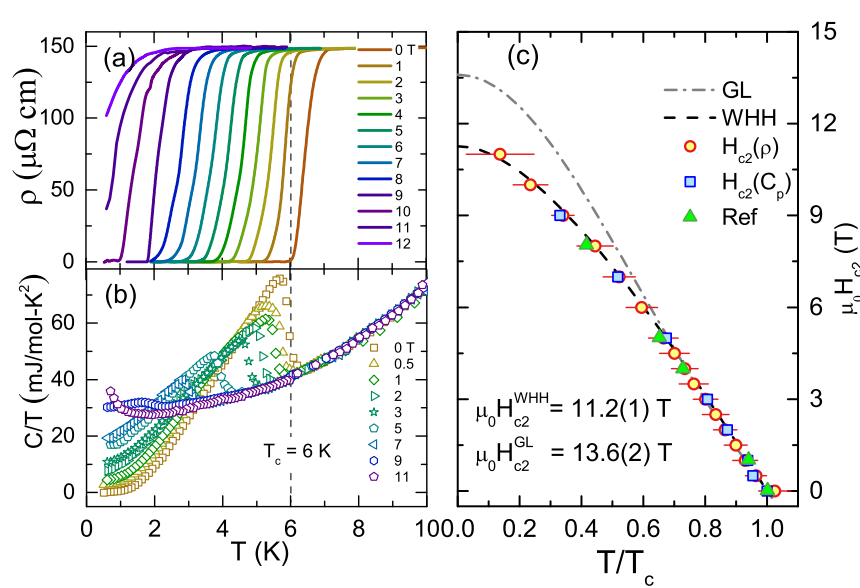

FIG. 2. (a) Temperature-dependent electrical resistivity and (b) specific heat at different applied magnetic fields up to $12 \mathrm{~T}$. From (c) the suppression of $T_{c}$ with an increasing field we determine an upper critical field $\mu_{0} H_{c 2}(0)=11.2(1) \mathrm{T}$. The dashed line represents a fit to the Werthamer-Helfand-Hohenberg (WHH) model without spin-orbit scattering, whereas the dashed-dotted line is a fit to the Ginzburg-Landau model (see the text).

$\mu_{0} H_{c 1}(T)=\mu_{0} H_{c 1}(0)\left[1-\left(T / T_{c}\right)^{2}\right]$, which provides a lower critical field $\mu_{0} H_{c 1}(0)=8.3(1) \mathrm{mT}$, consistent with the 8.4$\mathrm{mT}$ value calculated from magnetic penetration depth $\lambda(0)$. In the Ginzburg-Landau theory of superconductivity, the magnetic penetration depth $\lambda$ is related to the coherence length $\xi$ and the lower critical field $\mu_{0} H_{c 1}$ via $\mu_{0} H_{c 1}=$ $\left(\Phi_{0} / 4 \pi \lambda^{2}\right)[\ln (\kappa)+\alpha(\kappa)]$, where $\Phi_{0}=2.07 \times 10^{-3} \mathrm{~T} \mu \mathrm{m}^{2}$ is the quantum of magnetic flux, $\kappa=\lambda / \xi$ is the GinzburgLandau parameter, and $\alpha(\kappa)$ is a parameter which converges to 0.497 for $\kappa \gg 1$. By using $\mu_{0} H_{c 1}=8.3 \mathrm{mT}$ and $\xi=5.41 \mathrm{~nm}$ (calculated from $\mu_{0} H_{c 2}$ ), the resulting $\lambda(0)=286 \mathrm{~nm}$ is consistent with the experimental value from $\mu$ SR [see Fig. 3(c)]. With a Ginzburg-Landau parameter of $\kappa \sim 53 \gg 1, \mathrm{Re}_{24} \mathrm{Ti}_{5}$ is clearly a type-II superconductor. The temperature dependence of penetration depth $\lambda(T)$ can be estimated also from $\mu_{0} H_{c 1}(T)$ and $\xi(T)$, where $\xi(T)$ is related to the upper critical field $\mu_{0} H_{c 2}(T)=\Phi_{0} / 2 \pi \xi^{2}(T)$.

To investigate the behavior of the upper critical field $\mu_{0} H_{c 2}$, we measured the electrical resistivity $\rho(T)$ and specific-heat $C(T) / T$ at various magnetic fields. As shown in Figs. 2(a) and 2(b), the superconducting transition in both cases shifts towards lower temperatures upon increasing the magnetic field. Note that, for $\mu_{0} H=11 \mathrm{~T}$, the large upturn of specific heat at low $T$ is due to a Schottky anomaly from nuclear moments, which hides the superconducting transition. Similar features were also observed in other Re-based intermetallic superconductors [22,23]. The superconducting transition temperatures versus the normalized temperature $T / T_{c}$, as derived from both $\rho(T)$ and $C(T) / T$, are summarized in Fig. 2(c). Data taken from Ref. [18] are also plotted. The temperature dependence of the upper critical field $\mu_{0} H_{c 2}(T)$ was analyzed following the WHH model [24]. The dashed line in Fig. 2(c), a fit to the WHH model without considering spin-orbital scattering, gives $\mu_{0} H_{c 2}^{\mathrm{WHH}}(0)=11.2(1) \mathrm{T}$. The derived $\mu_{0} H_{c 2}(0)$ value is very close to the Pauli paramagnetic limit for the weak-coupling case $\mu_{0} H_{\mathrm{P}}=1.86 T_{c}=11.7(2) \mathrm{T}$, thus indicating the 

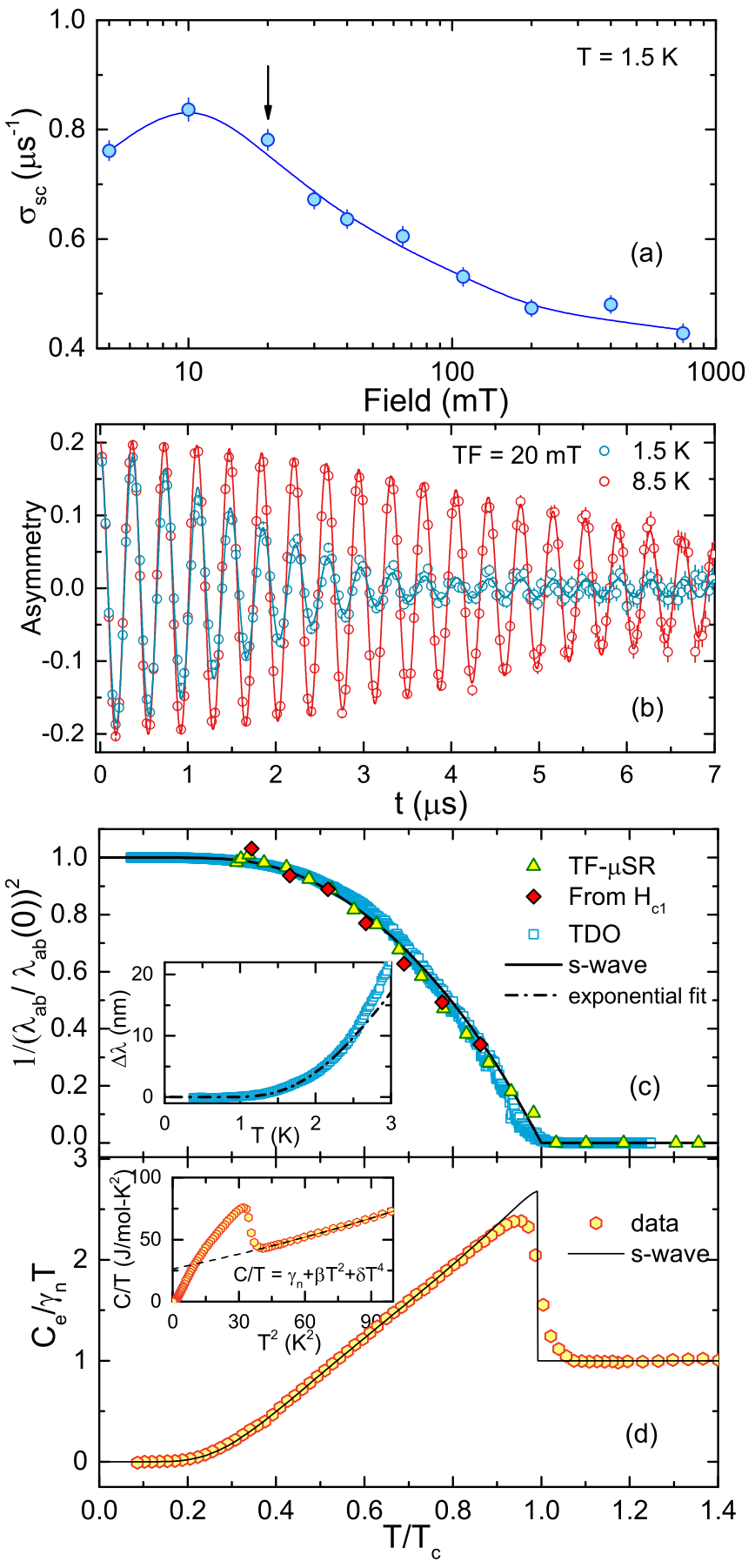

FIG. 3. (a) Field-dependent $\mu \mathrm{SR}$ relaxation rate at $T=1.5 \mathrm{~K}$. The arrow indicates the field used for the TF- $\mu$ SR studies of the superconducting phase. (b) Time-domain TF- $\mu$ SR spectra above and below $T_{c}$ show different relaxation rates. (c) Superfluid density versus temperature as determined from $\mu \mathrm{SR}$ (triangles), $H_{c 1}$ (diamonds) and TDO (squares) data. The inset shows the shift of penetration depth below $3 \mathrm{~K}$, and the dashed-dotted line indicates the exponential temperature dependence. (d) Zero-field electronic specific heat versus temperature. The inset: Raw $C / T$ data versus $T^{2}$. The dashed line is a fit to $C / T=\gamma_{\mathrm{n}}+\beta T^{2}+\delta T^{4}$ from which the phonon contribution was evaluated. The solid lines in (c) and (d) both represent fits using a fully gapped $s$-wave model. possibility of a singlet-triplet-mixing state. For completeness, we estimated the upper critical field also by means of the Ginzburg-Landau model $\mu_{0} H_{c 2}(T)=\mu_{0} H_{c 2}(0)\left(1-t^{2}\right) /(1+$ $t^{2}$ ), where $t=T / T_{c}$ is again the normalized temperature. As shown in Fig. 2(c) by a dashed-dotted line, at low fields, the fit is quite good. However, at higher applied fields, the fit deviates significantly from the data, providing an overestimated critical field value of $\mu_{0} H_{c 2}^{\mathrm{GL}}(0)=13.6(2) \mathrm{T}$. The remarkable agreement of the more elaborate WHH model with experimental data is clearly seen in Fig. 2(c).

To investigate the superconducting properties of $\mathrm{Re}_{24} \mathrm{Ti}_{5}$ on the microscopic level, we carried out TF- $\mu$ SR measurements in an applied field of $20 \mathrm{mT}$. The optimal field value for such experiments was determined via a preliminary field-dependent $\mu \mathrm{SR}$ depolarization-rate measurement at $1.5 \mathrm{~K}$. To avoid fluxpinning issues, the magnetic field (up to $750 \mathrm{mT}$ ) was applied in the normal state, and then the sample was cooled down to $1.5 \mathrm{~K}$. As shown in Fig. 3(a), the resulting Gaussian relaxation rate $\sigma_{\mathrm{sc}}$ versus the applied magnetic field exhibits a maximum near the lower critical field [see Fig. 1(c)]. By considering the decrease in intervortex distance with field and vortex-core effects, a field of $20 \mathrm{mT}$ (shown with an arrow), almost twice the $\mu_{0} H_{c 1}(0)$ value, was chosen for the temperature-dependent study.

Figure 3(b) shows two representative TF- $\mu$ SR spectra collected above and below $T_{c}$. Below $T_{c}$, the fast decay of muon-spin polarization reflects the inhomogeneous field distribution due to the FLL in the mixed superconducting state. The time-domain spectra were fitted by means of the following model with a Gaussian decay:

$$
A_{\mathrm{TF}}=A_{\mathrm{s}} \cos \left(\gamma_{\mu} B_{\mathrm{s}} t+\phi\right) e^{-\sigma^{2} t^{2} / 2}+A_{\mathrm{bg}} \cos \left(\gamma_{\mu} B_{\mathrm{bg}} t+\phi\right) .
$$

Here $A_{\mathrm{s}}$ and $A_{\mathrm{bg}}$ are the initial muon-spin asymmetries for muons implanted in the sample and sample holder, respectively, with the latter not undergoing any depolarization. $\gamma_{\mu}=2 \pi \times 135.53 \mathrm{MHz} / \mathrm{T}$ is the muon gyromagnetic ratio, $B_{\mathrm{s}}$ and $B_{\mathrm{bg}}$ are the local fields sensed by implanted muons in the sample and sample holder, $\phi$ is the (common) initial precession phase, and $\sigma$ is a Gaussian-relaxation rate. Given the nonmagnetic nature of the sample holder, $B_{\mathrm{bg}}$ practically coincides with the applied magnetic field and was used as an intrinsic reference.

In the superconducting state, the Gaussian-relaxation rate includes contributions from both the FLL $\left(\sigma_{\mathrm{sc}}\right)$ and the nuclear magnetic moments $\left(\sigma_{\mathrm{n}}\right)$. Since $\sigma_{\mathrm{n}}$ is expected to be temperature independent in the considered temperature range, the FLLrelated relaxation rate can be derived by subtracting the nuclear contribution from the measured Gaussian relaxation, i.e., $\sigma_{\mathrm{sc}}=$ $\sqrt{\sigma^{2}-\sigma_{\mathrm{n}}^{2}}$. Since $\sigma_{\mathrm{sc}}$ is directly related to the superfluid density $\left(\sigma_{\mathrm{sc}} \propto 1 / \lambda^{2}\right)$, the superconducting gap value and its symmetry can be determined from the temperature-dependent relaxation rate $\sigma_{\mathrm{sc}}(T)$. For small applied magnetic fields [in comparison with the upper critical field, i.e., $\left.H_{\text {appl }} / H_{c 2} \ll 1\right]$, the effective penetration depth $\lambda_{\text {eff }}$ can be calculated from $[25,26]$

$$
\frac{\sigma_{\mathrm{sc}}^{2}(T)}{\gamma_{\mu}^{2}}=0.00371 \frac{\Phi_{0}^{2}}{\lambda_{\mathrm{eff}}^{4}(T)} .
$$


In a polycrystalline sample, the effective penetration depth $\lambda_{\text {eff }}$ is usually determined by the shortest penetration depth $\lambda_{a b}$, the two being related via $\lambda_{\text {eff }}=3^{1 / 4} \lambda_{a b}$ [27]. Figure 3(c) shows the normalized superfluid density $\left(\rho_{\mathrm{sc}} \propto \lambda_{a b}^{-2}\right)$ as a function of temperature for $\operatorname{Re}_{24} \mathrm{Ti}_{5}$. The $\lambda_{a b}^{-2}$ data calculated from $\mu_{0} H_{c 1}$ and those from TDO measurements also are plotted, both clearly consistent with the $\mu \mathrm{SR}$ results. The temperature-dependent behavior of $\lambda_{a b}^{-2}$ is well described by an $s$-wave model with a single SC gap of about $1.08 \mathrm{meV}$ and a $\lambda(0)$ of $298 \mathrm{~nm}$. Such a superconducting gap is similar to that of other Re-based intermetallic superconductors, e.g., $\mathrm{Re}_{6} \mathrm{Zr}(1.21 \mathrm{meV})$ [14,28], $\mathrm{Re}_{6} \mathrm{Hf}(0.94 \mathrm{meV})$ [22,29], and $\mathrm{Re}_{24} \mathrm{Nb}_{5}(0.89 \mathrm{meV})$ [19]. Also the $2 \Delta / \mathrm{k}_{\mathrm{B}} T_{c}$ values of these compounds [e.g., 4.2(1) for $\mathrm{Re}_{24} \mathrm{Ti}_{5}$ ] are higher than 3.53, the value expected for a weakly coupled BCS superconductor, thus indicating moderately strong electron-phonon couplings in these materials. Moreover, the low-temperature penetration depth, shown in the inset of Fig. 3(c), exhibits an exponential temperature dependence, providing further evidence of fully gapped superconductivity in $\mathrm{Re}_{24} \mathrm{Ti}_{5}$.

Since the specific heat in the superconducting state also offers insight into the superconducting gap and its symmetry, the zero-field specific-heat data were further analyzed. The electronic specific-heat $\left(C_{\mathrm{e}} / T\right)$ is obtained by subtracting the phonon contribution from the experimental data. As shown in the inset of Fig. 3(d) by the dashed line, the normalstate specific heat is fitted with $C / T=\gamma_{\mathrm{n}}+\beta T^{2}+\delta T^{4}$. The derived $C_{\mathrm{e}} / T$ is then divided by the normal-state electronic specific-heat coefficient as shown in the main panel as a function of temperature. The solid line in Fig. 3(d) represents a fit with $\gamma_{\mathrm{n}}=26.4(2) \mathrm{mJ} \mathrm{mol}^{-1} \mathrm{~K}^{-2}$ and a single isotropic gap $\Delta(0)=1.9(1) k_{\mathrm{B}} T_{c}$. It reproduces very well the experimental data while being consistent with the TF- $\mu$ SR and TDO results [see Fig. 3(c)]. The ratio $\Delta C / \gamma T_{c}$ was found to be 1.4 , consistent with previous data [18] and in good agreement with the BCS-theory value of 1.43 .

To address the key question of the occurrence of timereversal symmetry breaking in $\mathrm{Re}_{24} \mathrm{Ti}_{5}$, we made use of ZF- $\mu$ SR. The large muon gyromagnetic ratio, combined with the availability of $100 \%$ spin-polarized muon beams, make $\mathrm{ZF}-\mu \mathrm{SR}$ a very powerful technique to detect the spontaneous fields as shown by its successful use in previous studies of $\mathrm{Re}_{6}(\mathrm{Zr}, \mathrm{Hf})[14,15], \mathrm{La}_{7} \mathrm{Ir}_{3}$ [16], $\mathrm{Sr}_{2} \mathrm{RuO}_{4}$ [21], and $\mathrm{PrOs}_{4} \mathrm{Sb}_{12}$ [30]. Normally, in the absence of external fields, the onset of the superconducting phase does not imply changes in the ZF muonspin-relaxation rate. However, in the case of TRS breaking, the onset of tiny spontaneous currents gives rise to associated (weak) magnetic fields, promptly detected by ZF- $\mu$ SR as an increase in the muon-spin-relaxation rate. Given the tiny size of such effects, we measured carefully the muon-spin-relaxation rate both well above $T_{c}$ and well inside the superconducting phase. As shown in Fig. 4(a), two representative ZF- $\mu$ SR spectra collected above $(8 \mathrm{~K})$ and below $(1.5 \mathrm{~K}) T_{c}$ show clear differences, especially at long times. To exclude the possibility of stray magnetic fields (which in any case would affect uniformly all data sets), the magnets were quenched before the measurements, and we made use of an active field-nulling facility. Without an external field, the relaxation is determined mostly by the nuclear magnetic moments, normally described by a Gaussian Kubo-Toyabe relaxation function $[31,32]$. A

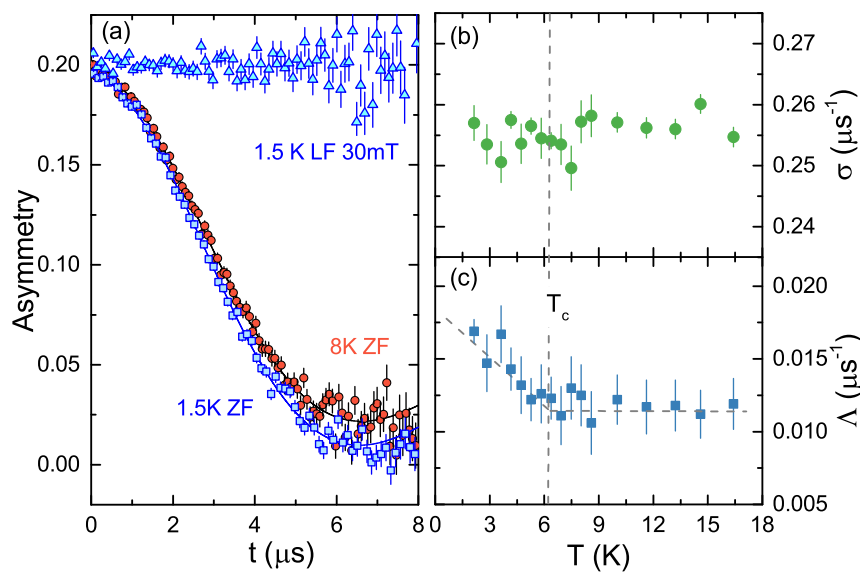

FIG. 4. (a) Representative zero-field $\mu \mathrm{SR}$ spectra for $\mathrm{Re}_{24} \mathrm{Ti}_{5}$ at 1.5 and $8 \mathrm{~K}$ and relevant fits by means of Eq. (3). A typical longitudinal-field (LF) LF- $\mu$ SR data set, collected at $1.5 \mathrm{~K}$ in a 30-mT longitudinal field, is also shown. (b) Temperature dependence of the nuclear relaxation rate $\sigma$, and (c) electronic relaxation rate $\Lambda$. Although $\sigma$ is almost temperature independent, $\Lambda$ shows a distinct increase below $T_{c}$.

possible spontaneous field contribution is accounted for by an additional exponential decay term. Consequently, the ZF- $\mu$ SR spectra could be fitted by means of a combined Lorentzian and Gaussian Kubo-Toyabe relaxation function,

$$
\begin{aligned}
A_{\mathrm{CKT}}= & A_{\mathrm{s}}\left\{\frac{1}{3}+\frac{2}{3}\left(1-\sigma^{2} t^{2}-\Lambda t\right)\right. \\
& \left.\times \exp \left(-\frac{\sigma^{2} t^{2}}{2}-\Lambda t\right)\right\}+A_{\mathrm{bg}} .
\end{aligned}
$$

Here $A_{\mathrm{s}}$ is the initial sample-related muon-spin asymmetry, whereas $A_{\text {bg }}$ represents a time- and temperature-independent background. As already shown in the TF- $\mu$ SR case (see Fig. 3), both the background and the nuclear contributions to the decay are independent of temperature. This is clearly the case also with ZF- $\mu$ SR [see Fig. 4(b)], where $\sigma(T)$ remains constant (within the experimental error) in the studied temperature range. On the other hand, the exponential component, related to the presence of spontaneous magnetic fields, shows a small yet distinct increase as the temperature is lowered below $T_{c}$ [see Fig. 4(c)].

Such an increase in $\Lambda(T)$, similar to that found also in $\operatorname{Re}_{6}(\mathrm{Zr}, \mathrm{Hf})[14,15]$, represents the signature of spontaneously occurring magnetic fields and of TRS breaking in the $\mathrm{Re}_{24} \mathrm{Ti}_{5}$ noncentrosymmetric superconductor. Given the small size of the considered effect, to rule out the possibility of an impurityinduced relaxation (typically relevant at low temperatures), we performed auxiliary LF- $\mu$ SR measurements at $1.5 \mathrm{~K}$. As shown in Fig. 4(a), a field of $30 \mathrm{mT}$ only is sufficient to lock the muon spins and to completely decouple them from the weak spontaneous magnetic fields, thus removing any relaxation traces related to them.

Up to now, several NCSCs, including $\mathrm{LaNiC}_{2}$ [13], $\operatorname{Re}_{6}(\mathrm{Zr}, \mathrm{Hf})[14,15]$, and $\mathrm{La}_{7}-\mathrm{Ir}_{3}$ [16] have been found to exhibit a TRS breaking in the superconducting state. Yet, in many others, as, e.g., $\mathrm{Mo}_{3} \mathrm{Al}_{2} \mathrm{C}$ [7], $\mathrm{Mg}_{10} \mathrm{Ir}_{19} \mathrm{~B}_{16}$ [33], $\mathrm{Re}_{3} \mathrm{~W}$ [34], and $\mathrm{PbTaSe}_{2}$ [35], the TRS is preserved. The $\mathrm{Re}_{24} \mathrm{Ti}_{5}$ 
considered here, a sister compound to $\operatorname{Re}_{6}(\mathrm{Zr}, \mathrm{Hf})$, is a new member of the TRS-breaking NCSCs, despite a relatively reduced ASOC. This strongly suggests that, although the presence of an ASOC seems essential to induce a TRS breaking in NSSCs, its strength is not a crucial condition. Indeed, although $\mathrm{LaNiC}_{2}$ [13] has a much weaker ASOC compared to $\mathrm{La}_{7} \mathrm{Ir}_{3}$ [16], the respective changes in zero-field muonrelaxation rates are comparable $\left(\Delta \Lambda \sim 0.01 \mu \mathrm{s}^{-1}\right)$. In our case, too, the replacement of the $5 d \mathrm{Hf}$ with the $3 d \mathrm{Ti}$, reduces remarkably the ASOC, yet the effects on TRS breaking remain comparable. Hence, we believe that TRS breaking in NSCSs is mostly related to the crystal-structure symmetry and, to test such a hypothesis, $\mathrm{La}_{7} \mathrm{~T}_{3}$ compounds $(T=$ transition metal, e.g., Ni, Pd, Rh, and Pt) represent good candidates since all of them exhibit a $\mathrm{Th}_{7} \mathrm{Fe}_{3}$-type crystal structure with the $3 d$ to $5 d$ transition metals covering a wide ASOC range.

The spin-triplet states can give rise to spontaneous fields in the superconducting state, which break the TRS. Most of these TRS-broken phases exhibit nodes in the superconducting gap, as e.g., $\mathrm{Sr}_{2} \mathrm{RuO}_{4}$ [21]. However, in highly symmetric systems, the TRS breaking can also occur in fully gapped states [36]. Thus, the cubic $\operatorname{Re}_{6}(\mathrm{Zr}, \mathrm{Hf})[14,15]$ and $\mathrm{Re}_{24} \mathrm{Ti}_{5}$ or the hexagonal $\mathrm{La}_{7} \mathrm{Ir}_{3}$ [16] all exhibit fully gapped superconducting states but with TRS breaking. A point-group analysis of $\operatorname{Re}_{6} \mathrm{Zr}$ [14] reveals that a mixed singlet and triplet state is allowed to break the TRS. The continuous search for other low-symmetry NCSCs provides a good opportunity to find non-s-wave superconductors with TRS breaking, hence, furthering our understanding of the NCSC physics.

To summarize, we investigated the noncentrosymmetric superconductor $\mathrm{Re}_{24} \mathrm{Ti}_{5}$ by means of $\mu \mathrm{SR}$ and TDO techniques. Bulk superconductivity with $T_{c}=6 \mathrm{~K}$ was characterized by magnetization, transport, and specific-heat measurements. The low-temperature penetration depth, superfluid density, and the zero-field specific-heat data reveal a nodeless superconductivity in $\mathrm{Re}_{24} \mathrm{Ti}_{5}$, well described by an isotropic $s$-wave model with a single gap. The spontaneous fields, which appear below $T_{c}$ and increase with decreasing temperature, provide strong evidence that the superconducting state of noncentrosymmetric $\mathrm{Re}_{24} \mathrm{Ti}_{5}$ breaks TRS and has an unconventional nature.

This work was supported by the National Key R\&D Program of China (Grants No. 2017YFA0303100 and No. 2016YFA0300202), the National Natural Science Foundation of China (Grant No. 11474251), and the Schweizerische Nationalfonds zur Förderung der Wissenschaftlichen Forschung (SNF).
[1] Non-Centrosymmetric Superconductors, edited by E. Bauuer and M. Sigrist (Springer-Verlag, Berlin, 2012), Vol. 847.

[2] M. Smidman, M. B. Salamon, H. Q. Yuan, and D. F. Agterberg, Superconductivity and spin-orbit coupling in noncentrosymmetric materials: A review, Rep. Prog. Phys. 80, 036501 (2017).

[3] I. Bonalde, W. Brämer-Escamilla, and E. Bauer, Evidence for Line Nodes in the Superconducting Energy Gap of Noncentrosymmetric $\mathrm{CePt}_{3} \mathrm{Si}$ from Magnetic Penetration Depth Measurements, Phys. Rev. Lett. 94, 207002 (2005).

[4] H. Mukuda, T. Fujii, T. Ohara, A. Harada, M. Yashima, Y. Kitaoka, Y. Okuda, R. Settai, and Y. Onuki, Enhancement of Superconducting Transition Temperature Due to the Strong Antiferromagnetic Spin Fluctuations in the Noncentrosymmetric Heavy-Fermion Superconductor CeIrSi ${ }_{3}$ : A ${ }^{29} \mathrm{Si}$ NMR Study under Pressure, Phys. Rev. Lett. 100, 107003 (2008).

[5] H. Q. Yuan, D. F. Agterberg, N. Hayashi, P. Badica, D. Vandervelde, K. Togano, M. Sigrist, and M. B. Salamon, Swave Spin-Triplet Order in Superconductors without Inversion Symmetry: $\mathrm{Li}_{2} \mathrm{Pd}_{3} \mathrm{~B}$ and $\mathrm{Li}_{2} \mathrm{Pt}_{3} \mathrm{~B}$, Phys. Rev. Lett. 97, 017006 (2006).

[6] M. Nishiyama, Y. Inada, and G.-Q. Zheng, Spin Triplet Superconducting State Due to Broken Inversion Symmetry in $\mathrm{Li}_{2} \mathrm{Pt}_{3} \mathrm{~B}$, Phys. Rev. Lett. 98, 047002 (2007).

[7] E. Bauer, G. Rogl, X.-Q. Chen, R. T. Khan, H. Michor, G. Hilscher, E. Royanian, K. Kumagai, D. Z. Li, Y. Y. Li, R. Podloucky, and P. Rogl, Unconventional superconducting phase in the weakly correlated noncentrosymmetric $\mathrm{Mo}_{3} \mathrm{Al}_{2} \mathrm{C}$ compound, Phys. Rev. B 82, 064511 (2010).

[8] J. Chen, L. Jiao, J. L. Zhang, Y. Chen, L. Yang, M. Nicklas, F. Steglich, and H. Q. Yuan, Evidence for two-gap superconductivity in the non-centrosymmetric compound $\mathrm{LaNiC}_{2}$, New J. Phys. 15, 053005 (2013).

[9] S. Kuroiwa, Y. Saura, J. Akimitsu, M. Hiraishi, M. Miyazaki, K. H. Satoh, S. Takeshita, and R. Kadono, Multigap Superconductivity in Sesquicarbides $\mathrm{La}_{2} \mathrm{C}_{3}$ and $\mathrm{Y}_{2} \mathrm{C}_{3}$, Phys. Rev. Lett. 100, 097002 (2008).

[10] E. Bauer, G. Hilscher, H. Michor, C. Paul, E. W. Scheidt, A. Gribanov, Y. Seropegin, H. Noël, M. Sigrist, and P. Rogl, Heavy Fermion Superconductivity and Magnetic order in Noncentrosymmetric $\mathrm{CePt}_{3} \mathrm{Si}$, Phys. Rev. Lett. 92, 027003 (2004).

[11] N. Kimura, K. Ito, H. Aoki, S. Uji, and T. Terashima, Extremely High Upper Critical Magnetic Field of the Noncentrosymmetric Heavy Fermion Superconductor CeRhSi ${ }_{3}$, Phys. Rev. Lett. 98, 197001 (2007).

[12] I. Sugitani, Y. Okuda, H. Shishido, T. Yamada, A. Thamizhavel, E. Yamamoto, T. D. Matsuda, Y. Haga, T. Takeuchi, R. Settai, and Y. Onuki, Pressure-induced heavy-fermion superconductivity in antiferromagnet $\mathrm{CeIrSi}_{3}$ without inversion symmetry, J. Phys. Soc. Jpn. 75, 043703 (2006).

[13] A. D. Hillier, J. Quintanilla, and R. Cywinski, Evidence for Time-Reversal Symmetry Breaking in the Noncentrosymmetric Superconductor $\mathrm{LaNiC}_{2}$, Phys. Rev. Lett. 102, 117007 (2009).

[14] R. P. Singh, A. D. Hillier, B. Mazidian, J. Quintanilla, J. F. Annett, D. McK. Paul, G. Balakrishnan, and M. R. Lees, Detection of Time-Reversal Symmetry Breaking in the Noncentrosymmetric Superconductor $\mathrm{Re}_{6} \mathrm{Zr}$ using Muon-Spin Spectroscopy, Phys. Rev. Lett. 112, 107002 (2014).

[15] D. Singh, J. A. T. Barker, A. Thamizhavel, D. McK. Paul, A. D. Hillier, and R. P. Singh, Time-reversal symmetry breaking in the noncentrosymmetric superconductor $\mathrm{Re}_{6} \mathrm{Hf}$ : Further evidence 
for unconventional behavior in the $\alpha$-Mn family of materials, Phys. Rev. B 96, 180501 (2017).

[16] J. A. T. Barker, D. Singh, A. Thamizhavel, A. D. Hillier, M. R. Lees, G. Balakrishnan, D. McK. Paul, and R. P. Singh, Unconventional Superconductivity in $\mathrm{La}_{7} \mathrm{Ir}_{3}$ Revealed by Muon Spin Relaxation: Introducing a New Family of Noncentrosymmetric Superconductor that Breaks Time-Reversal Symmetry, Phys. Rev. Lett. 115, 267001 (2015).

[17] B. T. Matthias, V. B. Compton, and E. Corenzwit, Some new superconducting compounds, J. Phys. Chem. Solids 19, 130 (1961).

[18] C. S. Lue, H. F. Liu, C. N. Kuo, P. S. Shih, J. Y. Lin, Y. K. Kuo, M. W. Chu, T. L. Hung, and Y. Y. Chen, Investigation of normal and superconducting states in noncentrosymmetric $\mathrm{Re}_{24} \mathrm{Ti}_{5}$, Supercond. Sci. Technol. 26, 055011 (2013).

[19] C. S. Lue, T. H. Su, H. F. Liu, and B.-L. Young, Evidence for $s$-wave superconductivity in noncentrosymmetric $\mathrm{Re}_{24} \mathrm{Nb}_{5}$ from ${ }^{93} \mathrm{Nb}$ NMR measurements, Phys. Rev. B 84, 052509 (2011).

[20] K. Matano, R. Yatagai, S. Maeda, and G.-Q. Zheng, Full-gap superconductivity in noncentrosymmetric $\operatorname{Re}_{6} \mathrm{Zr}, \mathrm{Re}_{27} \mathrm{Zr}_{5}$, and $\mathrm{Re}_{24} \mathrm{Zr}_{5}$, Phys. Rev. B 94, 214513 (2016).

[21] G. M. Luke, Y. Fudamoto, K. M. Kojima, M. I. Larkin, J. Merrin, B. Nachumi, Y. J. Uemura, Y. Maeno, Z. Q. Mao, Y. Mori, H. Nakamura, and M. Sigrist, Time-reversal symmetrybreaking superconductivity in $\mathrm{Sr}_{2} \mathrm{RuO}_{4}$, Nature (London) 394, 558 (1998).

[22] B. Chen, Y. Guo, H. Wang, Q. Su, Q. Mao, J. Du, Y. Zhou, J. Yang, and M. Fang, Superconductivity in the noncentrosymmetric compound $\mathrm{Re}_{6} \mathrm{Hf}$, Phys. Rev. B 94, 024518 (2016).

[23] J. Chen, L. Jiao, J. L. Zhang, Y. Chen, L. Yang, M. Nicklas, F. Steglich, and H. Q. Yuan, BCS-like superconductivity in the noncentrosymmetric compounds $\mathrm{Nb}_{x} \operatorname{Re}_{1-x}(0.13 \leqslant x \leqslant 0.38)$, Phys. Rev. B 88, 144510 (2013).

[24] N. R. Werthamer, E. Helfand, and P. C. Hohenberg, Temperature and purity dependence of the superconducting critical field, $H_{c 2}$. III. Electron spin and spin-orbit effects, Phys. Rev. 147, 295 (1966).

[25] W. Barford and J. M. F. Gunn, The theory of the measurement of the London penetration depth in uniaxial type II superconductors by muon spin rotation, Physica C 156, 515 (1988).
[26] E. H. Brandt, Properties of the ideal Ginzburg-Landau vortex lattice, Phys. Rev. B 68, 054506 (2003).

[27] V. I. Fesenko, V. N. Gorbunov, and V. P. Smilga, Analytical properties of muon polarization spectra in type-II superconductors and experimental data interpretation for mono- and polycrystalline HTSCs, Physica C 176, 551 (1991).

[28] D. A. Mayoh, J. A. T. Barker, R. P. Singh, G. Balakrishnan, D. McK. Paul, and M. R. Lees, Superconducting and normal-state properties of the noncentrosymmetric superconductor $\mathrm{Re}_{6} \mathrm{Zr}$, Phys. Rev. B 96, 064521 (2017).

[29] D. Singh, A. D. Hillier, A. Thamizhavel, and R. P. Singh, Superconducting properties of the noncentrosymmetric superconductor Re $_{6}$ Hf, Phys. Rev. B 94, 054515 (2016).

[30] Y. Aoki, A. Tsuchiya, T. Kanayama, S. R. Saha, H. Sugawara, H. Sato, W. Higemoto, A. Koda, K. Ohishi, K. Nishiyama, and R. Kadono, Time-Reversal Symmetry-Breaking Superconductivity in Heavy-Fermion $\mathrm{PrOs}_{4} \mathrm{Sb}_{12}$ Detected by Muon-Spin Relaxation, Phys. Rev. Lett. 91, 067003 (2003).

[31] R. Kubo and T. Toyabe, in Magnetic Resonance and Relaxation, edited by R. Blinc (North-Holland, Amsterdam, 1967).

[32] A. Yaouanc and P. D. de Réotier, Muon Spin Rotation, Relaxation, and Resonance: Applications to Condensed Matter (Oxford University Press, Oxford, 2011).

[33] A. A. Aczel, T. J. Williams, T. Goko, J. P. Carlo, W. Yu, Y. J. Uemura, T. Klimczuk, J. D. Thompson, R. J. Cava, and G. M. Luke, Muon spin rotation/relaxation measurements of the noncentrosymmetric superconductor $\mathrm{Mg}_{10} \mathrm{Ir}_{19} \mathrm{~B}_{16}$, Phys. Rev. B 82, 024520 (2010).

[34] P. K. Biswas, A. D. Hillier, M. R. Lees, and D. McK. Paul, Comparative study of the centrosymmetric and noncentrosymmetric superconducting phases of $\mathrm{Re}_{3} \mathrm{~W}$ using muon spin spectroscopy and heat capacity measurements, Phys. Rev. B 85, 134505 (2012).

[35] M. N. Wilson, A. M. Hallas, Y. Cai, S. Guo, Z. Gong, R. Sankar, F. C. Chou, Y. J. Uemura, and G. M. Luke, $\mu$ SR study of the noncentrosymmetric superconductor $\mathrm{PbTaSe}_{2}$, Phys. Rev. B 95, 224506 (2017).

[36] D. F. Agterberg, V. Barzykin, and L. P. Gor'kov, Conventional mechanisms for exotic superconductivity, Phys. Rev. B 60 , 14868 (1999). 